This item was submitted to Loughborough's Research Repository by the author.

Items in Figshare are protected by copyright, with all rights reserved, unless otherwise indicated.

\title{
Intragranular defects in As-deposited and cadmium chloride-treated polycrystalline cadmium telluride solar cells
}

PLEASE CITE THE PUBLISHED VERSION

https://doi.org/10.1109/PVSC.2016.7750295

PUBLISHER

IEEE

VERSION

AM (Accepted Manuscript)

\section{PUBLISHER STATEMENT}

Personal use of this material is permitted. Permission from IEEE must be obtained for all other uses, in any current or future media, including reprinting/republishing this material for advertising or promotional purposes, creating new collective works, for resale or redistribution to servers or lists, or reuse of any copyrighted component of this work in other works.

\section{LICENCE}

\section{All Rights Reserved}

\section{REPOSITORY RECORD}

Fiducia, Tom, Ali Abbas, Kurt Barth, Walajabad S. Sampath, and Michael Walls. 2016. "Intragranular Defects in As-deposited and Cadmium Chloride-treated Polycrystalline Cadmium Telluride Solar Cells". Loughborough University. https://hdl.handle.net/2134/16586345.v1. 


\title{
Intragranular Defects in As-Deposited and Cadmium Chloride-Treated Polycrystalline Cadmium Telluride Solar Cells
}

\author{
Thomas Fiducia ${ }^{1}$, Ali Abbas ${ }^{1}$, Kurt Barth ${ }^{2}$, Walajabad Sampath ${ }^{2}$ and Michael Walls ${ }^{1}$ \\ ${ }^{1}$ CREST (Centre for Renewable Energy Systems Technology), Loughborough University, Loughborough, United Kingdom \\ ${ }^{2}$ NSF I/UCRC for Next Generation Photovoltaics, Colorado State University, Fort Collins, United States
}

\begin{abstract}
Atomic-scale defects limit the open circuit Voltage, and the conversion efficiency of thin film polycrystalline cadmium telluride solar cells. Using state of the art aberrationcorrected high resolution transmission electron microscopy, the type, density and atomic structure of intragranular defects present in cadmium chloride treated and untreated CdTe has been established. The cadmium chloride activation process dramatically reduces defect density but faults do remain. Characterizing the defects in both materials is an essential first step to determining their potential electrical effects, and to understanding how the cadmium chloride treatment reduces their density. Improving our knowledge of the mechanisms involved can lead to further process improvements.
\end{abstract}

Index Terms-photovoltaic, thin-film, cadmium telluride, voltage, defect, TEM.

\section{INTRODUCTION}

The record cadmium telluride (CdTe) research cell efficiency is $21.0 \%$ [1]. Rapid improvements have been made recently after a period of stagnation at $16.7 \%$ [2]. This has enabled thin film CdTe modules to become one of the lowest cost sources of solar electricity [3]. However, the technology is still significantly short of its theoretical efficiency limit of $28-30 \%$ [4]. This is mainly due to a low open circuit Voltage $\left(V_{O C}\right)$, which is $76 \%$ of the thermodynamic limit for single junction CdTe devices [5]. This compares to figures of $97 \%$ for single crystal gallium arsenide and $85 \%$ for polycrystalline copper indium gallium diselenide [5]. While many factors can limit cell $V_{O C}$, device models have shown that simultaneously high carrier concentration and lifetime is necessary to obtain high open circuit Voltages in CdTe cells [6]. Defects that cause non-radiative recombination via deep level trap states can therefore be detrimental to cell voltages. While the most common planar defects are thought to be intrinsically benign, lacking dangling bonds [7], partial dislocations at the defect boundary are potentially electrically active [8]. In addition, point defects or $\mathrm{Cd}-\mathrm{Cd} / \mathrm{Te}-\mathrm{Te}$ wrongbonds that have been associated with planar defects may contribute to recombination [9]. Both planar and linear defects have been observed in untreated and treated material [10] [11], and so may be harming cell performance. Moreover, the marked increase in cell efficiency following $\mathrm{CdCl}_{2}$ treatment coincides with a drastic reduction in planar defect density [11]. While the improved efficiency could be down to other $\mathrm{CdCl}_{2}$ effects such as grain boundary passivation or stoichiometry improvements around twins [9], we believe the possible impact of intragranular defects warrants further investigation (intragranular defects are structurally extended defects that enter, reside within, or span the grain bulk). Here we use aberration-corrected High Resolution Transmission Electron Microscopy (HRTEM) to show the type, density, and atomic structure of intra-grain defects in $\mathrm{CdCl}_{2}$ treated and untreated material, and assess the potential electrical effects of the defects in both materials.

\section{Methodology}

Thin-film CdTe solar cells were fabricated using close space sublimation (CSS) in an all-in-one vacuum process as detailed in [12]. One of the samples was exposed to a $\mathrm{CdCl}_{2}$ activation treatment, while the second was left untreated. $\mathrm{CdCl}_{2}$ treatment was carried out at $388^{\circ} \mathrm{C}$ in an atmosphere of $2 \%$ oxygen. HRTEM samples were prepared by focused ion beam (FIB) milling using a dual beam FEI Nova 600 Nanolab. A standard in situ lift out method was used to prepare crosssectional samples through the coating into the glass substrate. A platinum over-layer was deposited to define the surface of the samples and homogenize the final thinning of the samples to electron transparency thicknesses [11]. Electron microscopy was carried out with a Jeol JEM-ARM200F TEM.

\section{RESULTS}

Perfect zinc blende (ZB) cadmium telluride consists of two interpenetrating face-centred cubic sublattices, one comprising cadmium $(\mathrm{Cd})$ atoms and the other tellurium (Te) atoms. One sublattice is translated from the other by one quarter of a body diagonal of the unit cell, forming a tetrahedral, diamondlike structure. In tetrahedral crystals, atoms can only occupy three sets of positions vertically above $\{111\}$ planes, labelled $\mathrm{A}, \mathrm{B}$ and $\mathrm{C}^{1}$ If successive pairs of $\{111\}$ layers are stacked such that their atoms occupy A positions, followed by B, and then $\mathrm{C}$ positions, repeating throughout the structure, then it is said to have the ideal ABC stacking sequence (zinc blende). Deviations from $\mathrm{ABC}$ stacking must, physically, involve a mirror reflection of atom positions across $\{111\}$ layers. This reflection has the effect of 'moving' atoms that would have been in the $\mathrm{C}$ positions, say, in an $\mathrm{ABC}$ sequence, to $\mathrm{A}$ positions, giving $\mathrm{A} \underline{\mathrm{B}} \mathrm{A}$ (where the underlined letter denotes a

\footnotetext{
${ }^{1}$ In binary crystals like CdTe a double-index notation is often used where lower case letters represent metal atoms (cadmium), and capitals represent non-metals (tellurium), giving an $\mathrm{AaBbCc}$ notation. For the present purposes $\mathrm{ABC}$ notation is sufficient as cadmium and tellurium layers of the same index (i.e. A and a) are always added or removed in pairs, so can be given a single denomination.
} 
mirror reflection about that letter/layer). A physical reflection in the stacking of layers is therefore accompanied by a reflection of the letters in the $\mathrm{ABC}$ notation. The arrangement, or spacing of reflections determines the kind of fault. For example a single reflection is called a twin (ABCBA) $)^{2}$ Two adjacent reflections in otherwise perfect material are called an intrinsic stacking fault. This is equivalent to the removal of a pair of $\{111\}$ layers from the stacking sequence, say A layers $(\mathrm{ABCBC})$. Two reflections separated by a non-reflection is called an extrinsic stacking fault. This is equivalent to the insertion of a pair of $\{111\}$ layers into the stacking sequence, say $B$ layers $(A B \underline{C B} \underline{A B C})$. A series of adjacent reflections (ABABA) is a different phase of CdTe called wurtzite (WZ), which is hexagonal rather than cubic. As twins and stacking faults involve reflections, they can be thought of as being made up of very thin wurtzite regions.

In order to determine the kinds of faults that are present in the treated and untreated materials, as well as their atomic structure and density, aberration-corrected HRTEM has been carried out on both materials. A detailed comparison of the defect properties of the two materials can therefore be made. Fig. 1 shows an aberration-corrected High Angle Annular Dark-Field (HAADF) image of a region of untreated CdTe absorber layer viewed down the $\langle 110\rangle$ direction. When viewed down this axis, adjacent cadmium and tellurium atomic columns appear as closely spaced intensity peaks, called dumbbells (see short white lines in the figure). The dumbbells lie parallel to one another along $\{111\}$ planes, which are observed edge-on in this viewing direction. When dumbbells remain parallel between adjacent $\{111\}$ layers, there is a continuation of normal $\mathrm{ABC}$ stacking. A mirror reflection of the dumbbell direction across $\{111\}$ layers indicates a reflection in the stacking of atoms, and so is a twin as described above. Twins are shown by the arrows on the right of Fig. 1. When twins are closely spaced like this, the assignment of faults as intrinsic, extrinsic, etc. is often ambiguous. In order to quantify the density of faults, it is therefore more appropriate to compare the number of dumbbell reflections (twins) to the number of non-reflections, where dumbbells remain parallel between adjacent layers. This can be done by defining a quantity called the twin density, which is the number of reflections per one hundred reflection opportunities, or twins per one hundred $\{111\}$ layers. For example the region shown in figure 1 has 20 reflections and 17 non-reflections, giving a twin density of 54. Including larger areas not shown, the number of reflections was 48 , and non-reflections 51 , a twin density of just over 48 . This is

\footnotetext{
${ }^{2}$ The name twin was initially only used for a single reflection surrounded by thick regions of perfect crystal on either side. Recently however [10] [9], the term has been used to describe any reflection in the $\{111\}$ stacking sequence, even if other faults exist nearby on either side of it. This might mean the mirror symmetry only extends a single $\{111\}$ layer either side of the reflection. Using this terminology stacking faults can therefore be described as being made up of multiple twins. To distinguish between the old and new meanings of the term, reflections with bulk perfect crystal on either side will be called 'conventional twins', and reflections just 'twins' for the remainder of the paper.
}

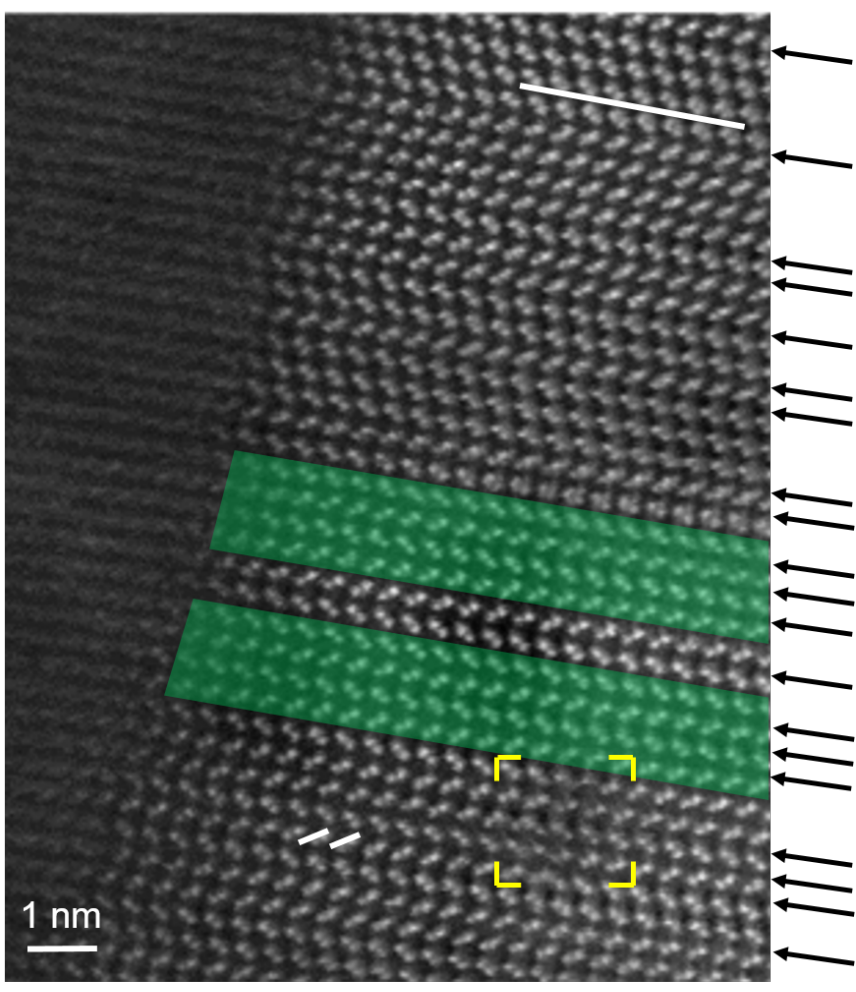

Fig. 1. Aberration-corrected HAADF image of untreated CdTe with the electron beam in the $\langle 110\rangle$ direction. The long white line represents the $\{111\}$ plane viewed edge-on. Arrows on the right designate twins. Thick wurtzite regions are indicated by the green shading and the yellow box highlights a group of partial dislocations.

essentially a $48 \%$ incidence of the wurtzite phase. Thicker WZ regions (defined here as three or more immediately adjacent reflections) are indicated by green shading in the figure. Although the region is highly faulted, the dumbbell positioning shows that tetrahedral bonding is conserved across the faults. This excludes a collection of mid-grain stacking fault terminations, indicated by the yellow box in the figure (the majority of the stacking faults span the grain bulk and terminate at the grain boundary). Distortion of the image in this area and the close proximity of the dislocations to one another make detailed characterisation difficult. However, it can be seen that these are Shockley-type partial dislocations as there is no extra or missing layer that would identify the faults as Frank-type. Partial dislocations necessarily contain non-tetrahedal bonding along the fault line. Inspection of the integrated intensity profiles of dumbbells in the region, in the manner of $\mathrm{Li}$ et al [10], shows that $\mathrm{Cd}-\mathrm{Te}$ dumbbell polarity is reversed across faults in the region, indicating normal $\mathrm{Cd}-\mathrm{Te}$ bonding.

Fig. 2 shows two grains of a cell that has undergone a $\mathrm{CdCl}_{2}$ treatment. Both grains are visible because one is rotated from the other about the viewing axis $\left(\mathrm{c} .90^{\circ}\right)$, keeping both sets of atomic columns parallel to the electron beam. This enables good visibility of the atomic configuration of the 


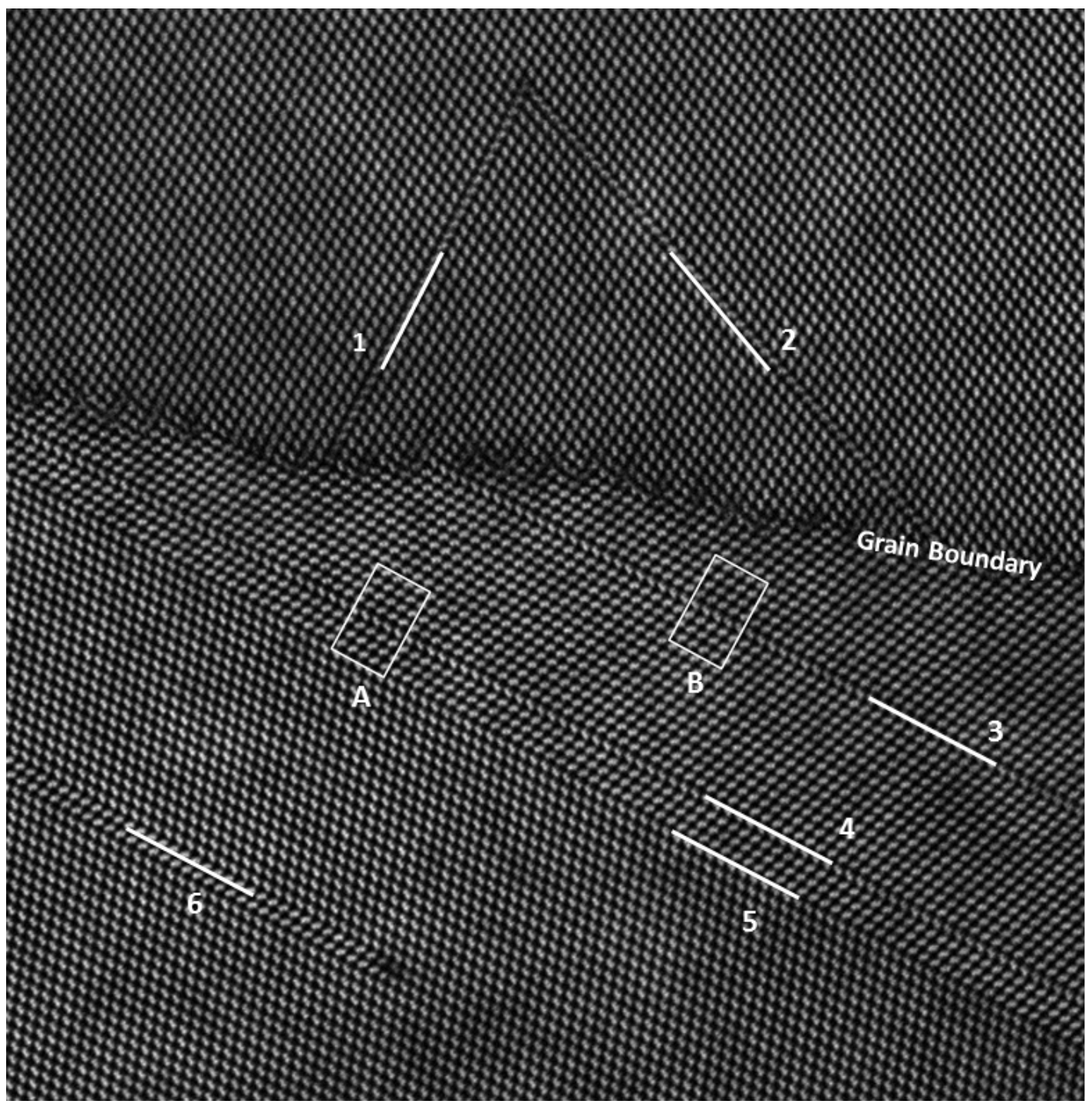

Fig. 2. Aberration-corrected HAADF image of a region of treated CdTe absorber layer viewed down the $<110>$ direction. Planar defects are labelled 1 to 6. Boxes A and B show equivalent regions of two intrinsic stacking faults. These are analysed in figures 3 and 4 respectively.

grain boundary. It can be seen that the fault density is much reduced compared to the untreated cell, with twin density in the lower grain at under 7 , compared to 48 in the untreated material. Intrinsic stacking faults (lines $3 \& 4$ ), a conventional twin boundary (5), and an extrinsic stacking fault (6) are seen in the bottom grain. The extrinsic SF can be seen to terminate within the grain. In the top grain, two intrinsic SFs terminate together forming a Lomer-Cottrell (acute) Stair-Rod partial dislocation.

Fig. 3 (i) shows a magnified image of part of stacking fault 4, corresponding to region $\mathrm{A}$ in Fig. 2. Cadmium and tellurium atomic columns can be clearly distinguished, enabling tetrahedron projections to be superimposed on top of the image (ii). These reveal that tetrahedral coordination is conserved across the stacking fault. ABC projection positions in (iii) show that pairs of $\{111\}$ layers follow the $\mathrm{ABCBC}$ stacking sequence of an intrinsic stacking fault.
Fig. 4 (i) shows a magnified image of part of stacking fault 3, corresponding to region B in Fig. 2. As intensity scales with the square of atomic number, the polarity of individual dumbbells can be determined by integrating pixel intensity values perpendicular to the length of a dumbbell (see arrows in (i) and profiles in (ii)). As tellurium has a higher atomic number than cadmium (52 versus 48 ), Te columns are identifiable as a higher peak in the intensity profile. Inspection of profiles in the image reveals a row of Te-Te wrong bonding along the top of the stacking fault. The dumbbell profiles below the stacking fault also suggest some wrong-bonding is present, however intensity differences are not as clear and consistent as those along the top of the fault (inconsistencies could be due to the presence of vacancies or strain in the lattice). 
A B C
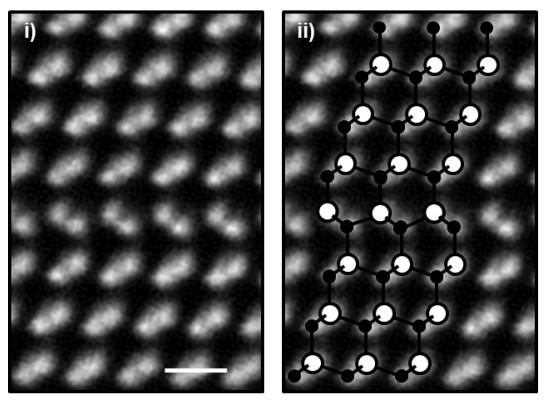

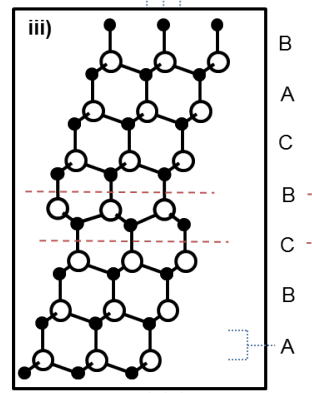

A B C

Fig. 3. (i) A magnified image of stacking fault 4. (ii) The same image with tetrahedron projections superimposed. (iii) Just the tetrahedrons from (ii). The letters on the side of the figure indicate the $\{111\}$ projection positions of the atoms in that pair of layers. The dotted red lines indicate mirror reflections in both the atom positions and the $\mathrm{ABC}$ notation.

\section{Discussion}

Once the defect structure of a material has been identified it is possible to begin to assess the likely effects of the faults on the material's electronic performance. Since modelling of electrical effects has already been done for many kinds of defect, for the same or a similar material system, an idea of their electrical effects can often be gathered from the literature without explicit calculation.

In our CSS-grown CdTe samples, the untreated material is essentially half wurtzite, with a twin as likely to form as a continuation of $\mathrm{ABC}$ stacking. This is interesting in itself as zinc blende is generally considered to be the stable phase of cadmium telluride [13]. Given the similarity in formation energy of the two phases however, the nearly equal preponderance should not come as a huge surprise [14]. Clearly, large sections of multiple grains would have to be inspected in order to obtain a statistical analysis of the tendency of one phase or the other to form within the cell.

The phase indecision in the untreated material results in a high density of tetrahedrally coordinated planar defects that in general extend across the entire grain, terminating at the grain boundary (this is known from lower resolution images). Tetrahedral planar faults are assumed to be benign as they only cause a negligible disruption in the lattice potential and a very small offset (order $0.01 \mathrm{eV}$ ) in the valence band maximum (VBM) [7]. While previous work has identified thicker buried wurtzite regions as potential hole traps [15], the VBM offset is again very small, and therefore unlikely to significantly affect electronic properties. With DFT calculations however, it is worth noting that faults are modelled in isolation. It is possible that close packing of defects like happens in the untreated material causes additional effects not picked up in the models. For example, closely spaced defects could cause a slight bumping of the valence band that might affect the movement of holes (although yet again the variation would

(i)
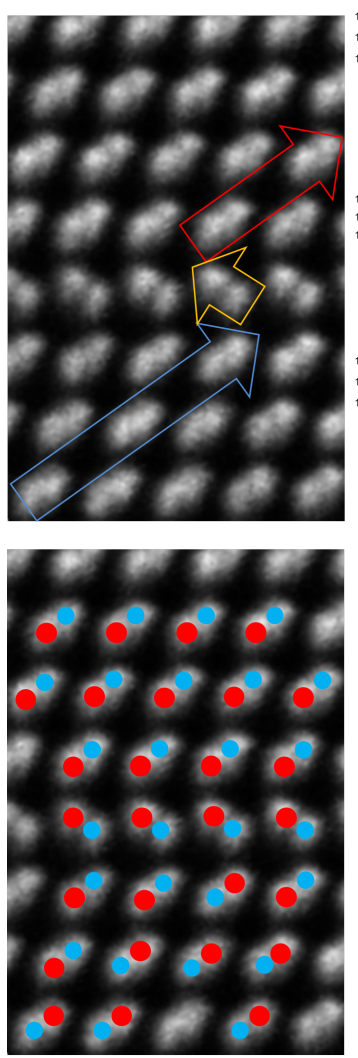

(iii) (ii)
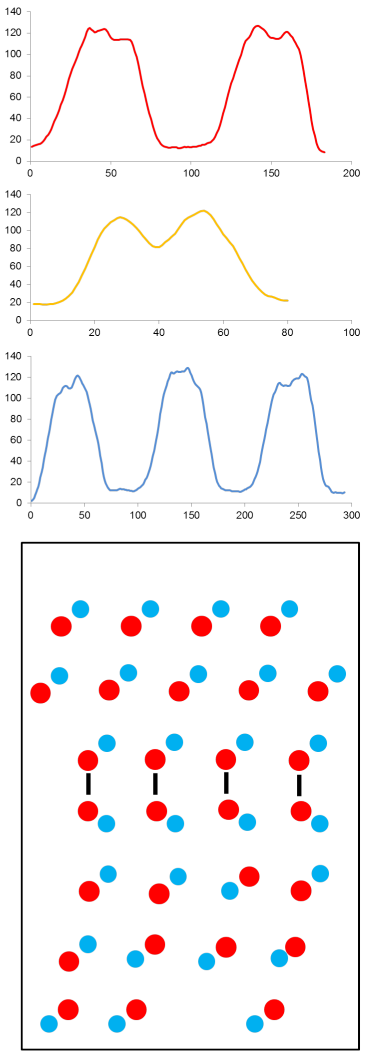

(iv)

Fig. 4. (i) Magnified image of stacking fault 3 at region B. Arrows show areas and directions along which integrated intensity profiles were taken. (ii) Intensity profiles from (i). Each curve is the profile from the corresponding arrow in (i). (iii) $\mathrm{Cd}$ and $\mathrm{Te}$ atomic column positions superimposed upon image (i), with red dots representing tellurium columns and blue dots representing cadmium columns. (iv) Just the column positions from (iii), with black lines showing a row of Te-Te wrong bonds.

be small, of the order of tens of meV [7] [15]). It is also not clear whether the very large areas of these kinds of faults in a typical grain 3 would lead to a greater, cumulative overall effect.

As the planar defects seen are intrinsically benign, any effects on recombination would have to result from wrongbonds or point defects associated with the faults. No significant wrong-bonding was seen in the region, so the only evidence of potentially harmful recombination caused by intra-grain defects in the untreated material is the presence of Shockley partial dislocations. Even with the partial dislocations however, there are reasons to believe that they may not be an issue in terms of performance. The first is that whether or not partial dislocations in CdTe are electrically active is not completely clear. While studies with silicon have shown that partial dislocations cause non-radiative recombination

\footnotetext{
${ }^{3}$ A cube-shaped grain with a side length of $1 \mu \mathrm{m}$ and a twin density of 50 , with $\{111\}$ layers parallel to one side of the cube, would contain around 1500 square microns of twin area, compared to a grain boundary area of 6 square microns.
} 
[8], DFT calculations on CdTe have concluded that certain partial dislocations, while containing dangling bonds, did not introduce localised states in the band gap [?]. The second is that the defects are linear in nature and have an apparently low density. As such, even if the defects are electrically active, it seems unlikely that their electrical effects would be significant compared to those of unpacified grain boundaries or point defects potentially associated with the twin boundaries.

Planar defect density is significantly reduced following $\mathrm{CdCl}_{2}$ treatment. In the limited areas analysed so far, the stacking faults that remain are tetrahedrally coordinated and so lack dangling bonds. The small area of wrong-bonding in region B may indicate a certain prevalence of electrically active wrong-bonding across stacking faults/twins in the material (Te-Te bonds have been shown to be electrically active [?]). However, it should be noted that bonding in this area could be affected by its proximity to the grain boundary. In order to establish the electrical activity of the fault terminations in the treated material, the exact defect type would have to be determined using Burgers circuit analysis, and this information used as the basis for DFT calculations. While Lomer-Cottrell stair-rod partial dislocations have been identified and their core structures determined in a recent paper by Paulauskas et al [16], no DFT has yet been done on these faults.

\section{CONCLUSION}

Aberration-corrected HRTEM has been carried out on asdeposited and $\mathrm{CdCl}_{2}$ treated $\mathrm{CdTe}$ absorber layers grown by CSS. The untreated material is found to be heavily faulted, with a twin density of 48 . Planar defects present in the material are tetrahedrally coordinated and show no significant wrong-bonding, so should be electrically benign (but for the possible presence of point defects). While there is some evidence of potentially harmful linear defects in the untreated material, densities are not high enough to suggest they are a primary cause of low efficiencies in as-deposited devices. Together these findings suggest the positive effect of the $\mathrm{CdCl}_{2}$ treatment on CdTe devices has more to do with its action on grain boundaries and point defects than on intragranular extended defects. Nevertheless, intra-grain defects remain in treated devices and may be contributing to non-radiative recombination. Further work is required to fully understand the relationship between intragranular planar defects, the presence of chlorine in the grain boundaries, and device efficiency.

\section{ACKNOWLEDGMENTS}

The Loughborogh authors are grateful to the UKERC Supergen SuperSolar Hub for funding. The authors at CSU are thankful for funding support from NSFs Accelerating Innovation Research and NSFs Industry/University Cooperative Research Center programs. TF is grateful for a studentship from the EPSRC CDT in New and Sustainable Photovoltaics.

\section{REFERENCES}

[1] M. A. Green, K. Emery, Y. Hishikawa, W. Warta, and E. D. Dunlop, "Solar cell efficiency tables (version 46)," Progress in Photovoltaics: Research and Applications, vol. 23, pp. 805-812, July 2015.

[2] M. Gloeckler, I. Sankin, and Z. Zhao, "CdTe Solar Cells at the Threshold to 20\% Efficiency," IEEE Journal of Photovoltaics, vol. 3, pp. 1389-1393, Oct. 2013.

[3] J. Balderelli, "Public Utilities Comission of Nevada Electronic Filing," tech. rep., 2015.

[4] W. Shockley and H. J. Queisser, "Detailed Balance Limit of Efficiency of p-n Junction Solar Cells," Journal of Applied Physics, vol. 32, no. 3, p. 510,1961

[5] R. M. Geisthardt, M. Topic, and J. R. Sites, "Status and Potential of CdTe Solar-Cell Efficiency," IEEE Journal of Photovoltaics, vol. 5, pp. 1217-1221, July 2015.

[6] J. Burst, J. Duenow, D. Albin, E. Colegrove, M. Reese, J. Aguiar, C.-S Jiang, M. Patel, M. Al-Jassim, D. Kuciauskas, S. Swain, T. Ablekim, K. Lynn, and W. Metzger, "CdTe solar cells with open-circuit voltage greater than 1 V," Nature Energy, no. February, p. in press, 2016.

[7] S.-h. Yoo, K. T. Butler, A. Soon, A. Abbas, J. M. Walls, and A. Walsh, "Identification of critical stacking faults in thin-film CdTe solar cells," Applied Physics Letters, vol. 105, p. 062104, Aug. 2014.

[8] D. Holt and B. Yacobi, Extended defects in semiconductors. 2007.

[9] T. Paulauskas, C. Buurma, E. Colegrove, Z. Guo, S. Sivananthan, M. K. Y. Chan, and R. F. Klie, "Atomic-resolution characterization of the effects of $\mathrm{CdCl} 2$ treatment on poly-crystalline CdTe thin films," Applied Physics Letters, vol. 105, no. 7, p. 071910, 2014.

[10] C. Li, J. Poplawsky, Y. Wu, A. R. Lupini, A. Mouti, D. N. Leonard, N. Paudel, K. Jones, W. Yin, M. Al-Jassim, Y. Yan, and S. J. Pennycook, "From atomic structure to photovoltaic properties in CdTe solar cells," Ultramicroscopy, vol. 134, pp. 113-125, Nov. 2013.

[11] A. Abbas, G. West, J. Bowers, P. M. Kaminski, B. Maniscalco, J. Walls, K. Barth, and W. Sampath, "Cadmium chloride assisted recrystallization of CdTe: The effect of annealing over-treatment," in 2014 IEEE 40th Photovoltaic Specialist Conference (PVSC), pp. 0701-0706, IEEE, June 2014.

[12] D. E. Swanson, J. M. Kephart, P. S. Kobyakov, K. Walters, K. C. Cameron, K. L. Barth, W. S. Sampath, J. Drayton, and J. R. Sites, "Single vacuum chamber with multiple close space sublimation sources to fabricate CdTe solar cells," Journal of Vacuum Science \& Technology A: Vacuum, Surfaces, and Films, vol. 34, p. 021202, Mar. 2016.

[13] R. T. Paul Siffert, "Introduction," in CdTe and Related Compounds; Physics, Defects, Hetero- and Nano-structures, Crystal Growth, Surfaces and Applications, Elsevier, 2009.

[14] S. Takeuchi and K. Suzuki, "Stacking Fault Energies of Tetrahedrally Coordinated Crystals," Physica Status Solidi (a), vol. 171, no. 1, pp. 99103, 1999.

[15] Y. Yan, M. M. Al-Jassim, K. M. Jones, S.-H. Wei, and S. B. Zhang, "Observation and first-principles calculation of buried wurtzite phases in zinc-blende CdTe thin films," Applied Physics Letters, vol. 77, no. 10, p. 1461,2000 .

[16] T. Paulauskas, C. Buurma, E. Colegrove, B. Stafford, Z. Guo, M. K. Y Chan, C. Sun, M. J. Kim, S. Sivananthan, and R. F. Klie, "Atomic scale study of polar LomerCottrell and Hirth lock dislocation cores in CdTe," Acta Crystallographica Section A Foundations and Advances, vol. 70, pp. 524-531, Nov. 2014. 